\title{
Universiteit
}

Leiden

The Netherlands

\section{Emotion regulation as mediator of treatment outcome in therapy for deliberate self-harm.}

Slee, N.; Spinhoven, P.; Garnefski, N.; Arensman, E.

\section{Citation}

Slee, N., Spinhoven, P., Garnefski, N., \& Arensman, E. (2008). Emotion regulation as mediator of treatment outcome in therapy for deliberate self-harm. Clinical Psychology And

Psychotherapy, 15, 205-216. Retrieved from https://hdl.handle.net/1887/14271

Version: $\quad$ Not Applicable (or Unknown)

License: $\quad$ Leiden University Non-exclusive license

Downloaded from: $\quad$ https://hdl.handle.net/1887/14271

Note: To cite this publication please use the final published version (if applicable). 


\title{
Emotion Regulation as Mediator of Treatment Outcome in Therapy for Deliberate Self-Harm
}

\author{
Nadja Slee, ${ }^{1 *}$ Philip Spinhoven, ${ }^{2}$ Nadia Garnefski ${ }^{1}$ and \\ Ella Arensman ${ }^{3}$ \\ ${ }^{1}$ Unit of Clinical Psychology, Institute of Psychological Research, Leiden \\ University, Wassenaarseweg, Leiden, the Netherlands \\ ${ }^{2}$ Unit of Clinical Psychology, and the Department of Psychiatry of the Leiden \\ University Medical Centre, Institute of Psychological Research, Leiden \\ University, Wassenaarseweg, Leiden, the Netherlands \\ ${ }^{3}$ National Suicide Research Foundation, Cork, Ireland
}

\begin{abstract}
This study presents the outcomes of mediator analyses as part of a randomized controlled trial of Cognitive-Behavioural Therapy (CBT) for young people who engage in deliberate self-harm (DSH). The study involved 90 people, aged 15-35 years, who were randomly assigned to CBT in addition to treatment as usual or to treatment as usual only. The findings showed that changes in DSH were partially mediated by changes in emotion-regulation difficulties, particularly difficulties with impulse control and goal-directed behaviours. In addition, the potential mediating role of symptoms of depression, anxiety and suicidal cognitions was examined. Although the CBT intervention significantly reduced depression, anxiety and suicidal cognitions, these measures of symptom severity did not play a mediating role. These findings suggest that interventions for DSH should not primarily focus on mental disorders associated with DSH, but should be DSH-specific and should target specific emotion-regulation difficulties. Copyright $\odot 2008$ John Wiley \& Sons, Ltd.
\end{abstract}

\section{INTRODUCTION}

Emotion regulation has a central role in theories of deliberate self-harm (DSH) (Linehan, 1993). The conceptualization of emotion regulation used here (see Gratz \& Roemer, 2004) emphasizes the functional nature of emotional responses, with emotion dysregulation referring to maladaptive responses to emotions. Specifically, Gratz and Roemer (2004) broadly define emotion regulation as the awareness, understanding and acceptance of emotions, as well as the ability to control behaviour in the context of emotional distress. Several lines of research have

*Correspondence to: Nadja Slee, Department of Clinical, Health and Neuropsychology, Wassenaarseweg 52, P.O. Box 9555, 2300 RB Leiden, The Netherlands.

E-mail: nadja.slee@fsw.leidenuniv.nl provided evidence consistent with the emotional dysregulation hypothesis for DSH. First, descriptive studies consistently show that compared with psychiatric and non-psychiatric control participants, DSH patients (often diagnosed with borderline personality disorder) score significantly higher on self-report measures tapping emotion regulation difficulties, including lower emotional awareness and clarity (Leible \& Snell, 2004; Levine, Marziali, \& Hood, 1997; Slee, Garnefski, Van der Leeden, Arensman, \& Spinhoven, 2008a), lower acceptance of emotions (Slee, Garnefski, Spinhoven, \& Arensman, 2008b) and difficulty controlling behaviour when experiencing negative emotions (Leible \& Snell, 2004; Slee et al., 2008b; Yen, Zlotnick, \& Costello, 2002). Furthermore, these patients make greater use of avoidant emotion regulation strategies (Bijttebier \& Vertommen, 1999). A limitation is that these studies rely on self-report measures 
only, which may have resulted in underreporting or over-reporting of emotion regulation difficulties. Second, an experimental investigation of distress tolerance among patients with borderline personality disorder revealed an unwillingness to experience unpleasant emotions (Gratz, Rosenthal, Tull, Lejuez, \& Gunderson, 2006), which is consistent with a recent theoretical model that describes the primary function of DSH as the avoidance of unpleasant emotions (Chapman, Gratz, \& Brown, 2006). However, this study involved a small and homogenous sample of participants without mood disorders, limiting the generalizability of the results. Further support for the emotion regulatory function of DSH comes from research among female members of a Dutch DSH support organization. This study shows that negative emotions are highest immediately before the episode of $\mathrm{DSH}$, drop markedly after and increase again one day after the episode of DSH, while the reversed pattern is observed for positive emotions (Kamphuis, Ruyling, \& Reijntjes, 2007). Although these findings are consistent with the emotional dysregulation hypothesis for DSH, the study sample is a self-selected group of support-seeking females with DSH and borderline personality disorder. In addition, the study draws on introspection and retrospective self-report. Finally, during a 24-hour naturalistic psychophysiological ambulatory monitoring approach, it was found that patients diagnosed with borderline personality disorder reported more negative emotions, fewer positive emotions and a greater intensity of negative emotions than healthy controls (Ebner-Priemer et al., 2007a). In a second study it was found that these patients, on average, took little me to fluctuate from a positive mood to a negative mood, which suggests affective instability (Ebner-Priemer et al., 2007b). Affective instability is the feature of borderline personality disorder most strongly related to DSH. However, Stone, Broderick, Shiffman, and Schwartz (2004) argue that the retrospective assessment of mood fluctuation, as demonstrated in the study of EbnerPriemer et al. (2007b), is problematic. Furthermore, the ambulatory monitoring studies only assess emotional experience and do not ask participants to report daily life stressors during the monitoring. As a result, we do not know if the participants exhibit heightened sensitivity to discrete stimuli and respond more intensively to events than to controls (as would follow from Linehan's theory of emotional dysregulation).

Cognitive behavioural therapy (CBT) for DSH is based on a theoretical model which assumes that vulnerability to DSH can be changed by changing emotion regulation deficits (Linehan, 1993; Slee, Arensman, Garnefski, \& Spinhoven, 2007). Treatment is directed at developing emotion regulation skills for coping with situations that trigger DSH, and modifying cognitions and behaviour that interfere with effective emotion regulation.

Randomized controlled trials (RCT's) of CBT for DSH are limited and their results are inconsistent. An RCT of brief CBT versus usual care showed that brief CBT was of limited efficacy in reducing DSH (Tyrer et al., 2003), whereas an RCT of cognitive therapy (CT) reported favourable outcomes with regard to the number of suicide attempts, depression severity and thoughts of hopelessness (Brown et al., 2005). The BOSCOT trial evaluated the clinical effectiveness and cost-effectiveness of CBT for suicidal patients with borderline personality disorder (Davidson et al., 2006; Palmer et al., 2006). Compared with patients in the TAU group, patients assigned to CBT were less likely to attempt suicide (Davidson et al., 2006). A RCT of schema-focused therapy among patients with borderline personality disorder also reported positive outcomes with regard to the number of episodes of DSH (Giesen-Bloo et al., 2006). To date, dialectical behavioural therapy is the only CBT intervention studied in more than one RCT, and has consistently been found to reduce self-injury in (female) patients with borderline personality disorder (Linehan et al., 2006). Furthermore, a recent RCT showed that DSH patients who received CBT in addition to usual care had significantly greater reductions in DSH, suicidal cognitions, symptoms of depression and anxiety and significantly greater improvements in self-esteem and problem-solving ability than controls (Slee et al., 2008a).

However, as the evidence supporting the efficacy of CBT for DSH accumulates, there are no studies investigating the mechanism of action of CBT. Understanding the mechanisms through which CBT operates would advance the development of innovative treatment strategies. Identifying mechanisms of change promises not only to improve treatment but also to enhance understanding of the nature of DSH. If a treatment has its effects by influencing a particular process, this finding points to the importance of this process in the maintenance of the disorder. Analysing these mechanisms therefore also provides an indirect test of the theoretical model of mechanisms maintaining the disorder.

On the basis of contemporary psychological theories of DSH, it makes sense to ask whether 
the changes resulting from CBT are mediated by changes in emotion-regulation difficulties. The main objective of the present study was to investigate whether changes in specific emotionregulation difficulties in DSH patients treated with CBT indeed mediated treatment outcome.

Considering the targets of CBT, it was expected that difficulties with impulse control, difficulties with goal-directed behaviours, lack of awareness of emotions and non-acceptance of emotions would be the most important mediators of treatment outcome. Few empirical studies have examined the association between particular aspects of emotion regulation difficulties and DSH. One study found that none of the correlations between $\mathrm{DSH}$ and aspects of emotion regulation differed significantly from one another (Gratz \& Roemer, 2004). However, this study involved a non-clinical population of college students. In another study, a clinical group of young women who harmed themselves was compared with a group of young women without a history of DSH across various aspects of emotion regulation. Controlling for depression severity, non-acceptance of emotions independently predicted DSH (Slee et al., 2008b). Given the sparse empirical evidence on particular emotion-regulation difficulties and DSH, our expectations of mediation are largely based on the targets of CBT. We expected four aspects of emotion regulation to mediate treatment change: difficulties with impulse control, difficulties engaging in goal-directed behaviours, lack of awareness of emotions and non-acceptance of emotions. In addition to these specific hypotheses, we explored the possible mediating role of lack of clarity of emotions and of limited access to emotionregulation strategies. Finally, we investigated the possible mediating role of symptom severity (depression, anxiety and suicidal cognitions).

The hypothesis that the effects of CBT on DSH would be mediated by changes in specific emotionregulation difficulties was tested in accordance with the approach advocated by Baron and Kenny (1986). According to Baron and Kenny, a variable may be called a mediator 'to the extent that it accounts for the relation between the predictor and the criterion' (Baron \& Kenny, 1986, pp. 1176). Figure 1 illustrates the Baron and Kenny approach. The first model shows the direct effect of the treatment condition on DSH (C). The second model represents mediation: $C^{\prime}$ is the direct effect of the treatment condition on DSH after controlling for the mediator. The results reported here are from a clinical trial that examined the outcome of CBT on
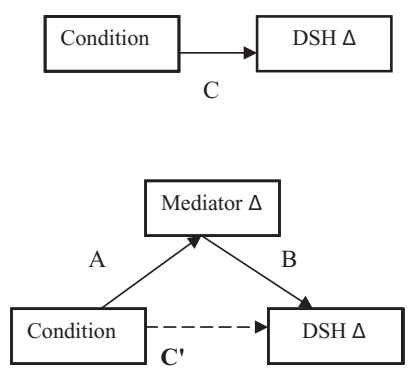

Figure 1. Model of mediated intervention effects. Dotted lines indicate that the mediator variable was controlled for. $\Delta=$ change

DSH (Slee et al., 2008a). We perform the mediator analyses using the baseline and 9-month followup data from this trial in order to test whether change in specific emotion regulation difficulties predicts reduction of DSH at 9-months follow-up in those who received CBT in addition to treatment as usual (TAU) compared with TAU only.

\section{METHOD}

\section{Patients and Procedure}

In order to be eligible, patients had to meet the following criteria: having recently engaged in DSH and being aged 15-35 years old. Patients were excluded if they reported severe psychiatric disorders requiring intensive inpatient treatment, were unable to converse in Dutch, had cognitive impairments or lived outside the region of Leiden.

Participants were recruited from March 2003 through April 2006 at the Leiden University Medical Centre and the local Mental Health Centre Rivierduinen. Those who agreed to participate and who were found to be eligible for the study $(n=$ 90) were randomly assigned to 12 sessions of CBT in addition to TAU $(n=48)$, or to TAU only $(n=$ 42). These participants were invited for subsequent assessments 3, 6 and 9 months following the baseline interview. For the present article, the baseline and 9-month follow-up measurements were used.

Our analyses were based on the intent-to-treat sample $(n=90)$. At baseline, there were no missing data. At 9-months follow-up, data were missing for 17 patients, all dropouts. Of the 17 dropouts, eight had dropped out right after the baseline interview and nine patients had dropped out during the course of the study. For each individual, missing values were replaced by the last observed value of that variable (using the so-called Last Observation Carried 
Forward (LOCF) method). To control for the impact of missing data and the use of the LOCF method on the mediation analyses, we performed the analyses both in the intent-to-treat sample $(n=90)$ and in the completers sample $(n=73)$. The completers sample consisted of the sample of 90 patients minus the eight early dropouts and the nine patients who dropped out during the course of the study. A complete description of the flow of participants through the study is given elsewhere (Slee et al., 2008a).

\section{Measures}

\section{Demographics}

During a structured clinical interview, demographic information was obtained regarding age, gender, living situation, marital status, educational level, job status and nationality.

\section{Outcome: DSH}

The primary outcome measure of the study was the number of episodes of DSH in the past 3 months, which was assessed using a structured clinical interview. DSH was defined as including both deliberate self-poisoning (overdose) and deliberate self-injury (Hawton, Zahl, \& Weatherall, 2003). An overdose was defined as the deliberate ingestion of more than the prescribed or recommended amount of chemical substances with the intention of self-harm. Patients were also asked about incidents of deliberate self-injury, which was defined as intentional self-injury, irrespective of the apparent purpose of the act, and included cutting, scratching, punching, kicking and headbanging. In this definition, both the original parasuicide definition of the WHO/Euro study and their current nomenclature of fatal and non-fatal suicidal behaviour is included, as well as habitual behaviours and self-injuries with no intent to die, which they exclude (De Leo, Bille-Brahe, Kerkhof, \& Schmidtke, 2004; Schmidtke, Bille-Brache, Leo, \& Kerkhof, 2004). Thus, all behaviour that was selfinitiated with the intent to harm the body (regardless of intent to die) is included. The study does not distinguish between suicidal acts and self-injury.

\section{Emotion Regulation Difficulties as Proposed Mediators of Treatment Outcome}

The Difficulties in Emotion Regulation Questionnaire (DERS) (Gratz \& Roemer, 2004) contains six dimensions of emotion regulation wherein difficulties may occur, including (a) lack of awareness of emotional responses (e.g., 'I pay attention to how
I feel' = reverse-scored item), (b) lack of clarity of emotional responses (e.g., 'I have difficulty making sense out of my feelings'), (c) non-acceptance of emotional responses (e.g., 'When I'm upset, I feel ashamed with myself for feeling this way'), (d) limited access to emotion regulation strategies perceived as effective (e.g., 'When I'm upset, I believe that there is nothing I can do to make myself feel better'), (e) difficulties controlling impulses when experiencing negative emotions (e.g., 'When I'm upset, I feel out of control'), and (f) difficulties engaging in goal-directed behaviours when experiencing negative emotions (e.g., 'When I'm upset, I have difficulty concentrating'). All questions are self-rated from 1 (almost) never to 5 (almost) always. Scores on the subscales range from 5-25 ('Clarity', 'Goals'), from 6-30 ('Awareness', 'Nonacceptance') and from 7-35 ('Impulses', 'Strategies'). All of the DERS subscales have adequate internal consistency, with alpha reliabilities of 80 or higher for each subscale (Gratz \& Roemer, 2004). In this study we also found alpha reliabilities of 0.80 or higher for each subscale: 0.82 for lack of awareness, 0.87 for lack of clarity, 0.83 for non-acceptance, 0.85 for limited strategies, 0.90 for difficulties controlling impulses, and 0.80 for difficulties goals.

\section{Symptom Severity (Depression, Anxiety and Suicidal Cognitions) as Proposed Mediators of Treatment Outcome}

Depression was measured by the Beck Depression Inventory II (BDI-II) (Beck, Steer, \& Brown, 1996a): a 21-question depression scale with each answer rated $0-3$. Scores range from 0-63. The test has high internal consistency with an alpha reliability of 0.91 (Beck et al., 1996a; Beck, Steer, Ball, \& Ranieri, 1996b). In this study we found an alpha reliability of 0.93 .

Anxiety was measured by the Symptom Checklist-90 (Arindell \& Ettema, 1986; Derogatis, Lipman, \& Covi, 1973), which is a self-report clinical rating scale of psychiatric symptomatology. It consists of 90 items in total, with 10 items for the subscale anxiety, five-point Likert, ranging from 'not at all distressing' (0) to 'extremely distressing' (4). Scores of this subscale range from 0-40. Previous studies have reported alpha-coefficients ranging from 0.71 to 0.91 for the anxiety subscale. In addition, test-retest reliabilities were found to be good and the subscale showed strong convergent validity with other conceptually related scales (Arrindell \& Ettema, 1986). In this study we found an alpha reliability of 0.93 for the anxiety subscale. 
Suicidal cognitions were measured by the Suicide Cognition Scale (Rudd, Joiner, \& Rajab, 2001): 20 questions about core beliefs of perceived burdensomeness ('I am a burden to my family'), helplessness ('No one can help solve my problems'), unlovability ('I am completely unworthy of love') and poor distress tolerance ('When I get this upset, it is unbearable'), with each answer rated 1 (strongly disagree) to 5 (strongly agree). Scores on the total scale range from 20-100. Scores on the subscale Perceived Burdensomeness (two items) range from 2-10, scores on the subscale Helplessness (five items) range from $5-25$, scores on the subscale Unlovability (six items) range from 6-30 and scores on the subscale Poor Distress Tolerance (seven items) range from 7-35. In this study we found an alpha reliability of 0.96 for the total scale, of 0.74 for Perceived Burdensomeness, of 0.88 for Helplessness, of 0.90 for Poor Distress Tolerance and of 0.89 for Unlovability.

\section{Treatment Interventions}

\section{Cognitive-Behavioural Therapy Intervention (CBT-condition)}

In addition to usual care (e.g., psychotropic medication, psychotherapy or psychiatric hospitalizations), participants in the CBT condition were to receive 12 outpatient CBT sessions specifically developed for preventing repeated DSH. Ten out of 12 sessions were given weekly; the last two sessions were follow-up sessions. Altogether, the intervention lasted 5.5 months. The central feature of this intervention was the identification and modification of the mechanisms that maintained the individual's self-harming behaviour. Thus, the treatment started with the assessment of the most recent episode of DSH. The therapist then specified how emotional, cognitive and behavioural factors played a role in the maintenance of DSH. Specific maintenance factors that were addressed included dysfunctional cognitions, emotion regulation difficulties and poor problem solving. Changing emotion regulation difficulties involved interventions geared towards mindfulness, acceptance and exposure with response prevention. Towards the end of therapy, relapse prevention was addressed as well. A manual was written to standardize the intervention (available on request).

All therapists were experienced practitioners of CBT and were experienced in working with patients who engage in DSH. Prior to taking part in the research project, they received two days of training in the standardized protocol. At monthly meetings, the treatment sessions were reviewed and therapists could share their experiences with their colleagues.

\section{Treatment as Usual (TAU) Comparison Condition}

For ethical reasons, participants in this group were free to pursue any treatment they deemed warranted. Most of the interventions involved a limited number (between 2 and 30) of sessions of individual psychotherapy such as CBT and interpersonal psychotherapy. Social skills training was also common, especially among adolescents and young adults. No treatment specific to DSH was reported. These treatments focused on specific psychiatric problems (e.g., depression) or on other needs of the patient (e.g., problems with housing, finances, social isolation). In addition to psychosocial interventions, the majority of the patients received psychotropic medication. The number of psychiatric hospitalizations was also recorded.

\section{Data Analyses}

We examined baseline differences between patients in CBT and TAU in demographic characteristics, DSH, symptom severity, and emotion-regulation difficulties using $t$-test analyses for continuous variables and chi-square tests for categorical variables. In addition, we examined differences in DSH, symptom severity and emotion regulation during the 9-month follow-up between patients in CBT and TAU, using $t$-tests. Pearson correlations were calculated to examine the relationships between the subscales of emotion regulation difficulties and DSH, as well as symptom severity and DSH.

The hypothesis that the effects of CBT on DSH would be mediated by changes in specific emotion regulation difficulties was tested in accordance with approach advocated by Baron and Kenny (1986). Baron and Kenny (1986) proposed a fourstep approach in which several regression analyses are conducted. First, treatment condition should predict change in the outcome (Path C). Second, treatment condition should predict change in the proposed mediator (Path A). Third, change in the mediator should be significantly associated with change in the outcome in the CBT condition (Path B). Fourth, the effect of treatment condition on change in the outcome should be attenuated when change in the mediator is statistically controlled (Path $C^{\prime}$ ) (see Figure 1). Separate linear regression analyses were used to examine mediation. In these 
analyses, corrections were made for the baseline scores of DSH and the proposed mediators by calculating residualized change scores. Residualized change scores are the 9-month follow-up scores after statistically correcting for any baseline differences on this measure using linear regression analysis.

\section{RESULTS}

\section{Group Comparisons at Baseline and 9-Month Follow-up}

Of the 90 patients, $93 \%$ were female. Their mean age was 24.2 years (standard deviation $[\mathrm{SD}]=5.6$ ), $92 \%$ were of Dutch nationality, $71 \%$ lived alone and $74 \%$ were unmarried. In addition, $29 \%$ went to school or studied, $24 \%$ had a full-time or parttime job and $38 \%$ lived on social welfare benefits. There were no significant differences between the CBT condition and TAU condition with regard to these demographics.

Table 1 contains the means and SDs for DSH, symptom severity and emotion regulation difficulties in both groups at baseline and 9-month follow-up. $t$-Tests showed no significant baseline differences on DSH, measures of symptom severity and measures of emotion regulation difficulties, except for lack of awareness of emotions, which was significantly more often reported in CBT than in TAU $(t=2.99$, degree of freedom $[\mathrm{df}]=88$, $p=0.004$ ) (see Table 1). The baseline difference in lack of awareness of emotions was controlled for at the 9-month follow-up. Furthermore, at 9month follow-up, significant group differences were found for all study variables, with patients in CBT reporting significantly lower scores of DSH, measures of emotion regulation difficulties and measures of symptom severity (see Table 1).

\section{Pearson Correlations between DSH and Emotion-Regulation Difficulties at Baseline}

Correlations between subscales ranged between 0.01 (lack of clarity and DSH) and 0.72 (difficulties with impulse control and difficulties with goaldirected behaviours) (see Table 2). Contrary to our expectations, positive correlations were found for DSH and only half of the DERS-subscales: lack of awareness, limited access to strategies and difficulties with goal-directed behaviours.

\section{Pearson Correlations between DSH and Symptom-Severity Measures at Baseline}

Correlations between subscales ranged between 0.19 (DSH and suicidal cognitions) and 0.74 (depression and suicidal cognitions) (see Table 3).

Table 1. Means and standard deviations (SDs) at baseline and 9-month follow-up for the treatment outcome and proposed mediators

\begin{tabular}{|c|c|c|c|c|}
\hline Variable & $\begin{array}{l}\text { CBT }(n=42) \\
\text { Baseline } \\
\text { Mean (SD) }\end{array}$ & $\begin{array}{l}\text { TAU }(n=48) \\
\text { Mean }(S D)\end{array}$ & $\begin{array}{c}\text { CBT }(n=42) \\
9 \text { month follow-up } \\
\text { Mean (SD) }\end{array}$ & $\begin{array}{l}\text { TAU }(n=48) \\
\text { Mean }(S D)\end{array}$ \\
\hline \multicolumn{5}{|l|}{ Outcome } \\
\hline DSH & $14.63(10.49)$ & $11.32(11.34)$ & $2.06(6.04)$ & $5.39(8.70)^{*}$ \\
\hline \multicolumn{5}{|c|}{ Proposed mediators: emotion regulation difficulties (DERS subscales) } \\
\hline Lack of awareness of emotions & $21.39(4.60)$ & $18.34(5.07)^{*}$ & $16.67(5.91)$ & $19.12(4.33)^{*}$ \\
\hline Lack of clarity of emotions & $17.59(4.02)$ & $16.71(5.24)$ & $12.98(5.21)$ & $16.20(4.58)^{*}$ \\
\hline Non-acceptance of emotions & $20.92(5.04)$ & $20.88(5.18)$ & $14.37(6.17)$ & $19.49(6.17)^{* *}$ \\
\hline Limited strategies & $22.63(5.60)$ & $23.59(6.03)$ & $15.98(6.85)$ & $22.78(7.50)^{* *}$ \\
\hline Difficulties impulse control & $25.45(6.11)$ & $26.56(6.80)$ & $17.98(8.51)$ & $25.41(8.17)^{* *}$ \\
\hline Difficulties goal-directed behaviours & $19.41(3.54)$ & $19.76(3.65)$ & $14.82(5.52$ & $19.02(4.63)^{* *}$ \\
\hline \multicolumn{5}{|l|}{ Proposed mediators: symptom severity } \\
\hline Depression (BDI-II) & $32.53(16.13)$ & $34.24(13.97)$ & $14.33(13.70)$ & $30.12(16.27)^{* *}$ \\
\hline Anxiety (SCL-90) & $29.84(8.51)$ & $28.27(10.55)$ & $20.65(7.94)$ & $28.02(10.57)^{* *}$ \\
\hline Suicidal cognitions (SCS) & $58.61(14.12)$ & $62.90(19.56)$ & $40.29(18.61)$ & $56.76(18.98)^{* *}$ \\
\hline
\end{tabular}

${ }^{*} \mathrm{p}<0.05 ;{ }^{* *} \mathrm{p}<0.01 ;{ }^{* * *} \mathrm{p}<0.001$.

DSH = deliberate self-harm. CBT = cognitive behavioural therapy. TAU = therapy as usual. BDI-II = Beck Depression Inventory II. DERS $=$ Difficulties in Emotion Regulation Questionnaire.

SCL-90 = Symptom Checklist 90 . 
Table 2. Pearson correlations between the number of episodes of DSH at baseline and emotion regulation difficulties in c patients $(n=90)$

\begin{tabular}{lccccccc}
\hline & DSH & Aware & Clarity & Non-accept & Strategies & Impulses & Goals \\
\hline DSH & - & $0.29^{*}$ & 0.01 & 19 & $0.23^{*}$ & 0.10 & $0.17^{* *}$ \\
Lack of awareness of emotions & & - & $0.35^{* *}$ & 0.19 & 0.10 & 0.08 & 0.05 \\
Lack of clarity of emotions & & & - & $0.34^{* *}$ & $0.25^{*}$ & $0.53^{* *}$ & $0.26^{*}$ \\
Non-acceptance of emotions & & & & - & $0.48^{* *}$ & $0.40^{* *}$ & $0.48^{* *}$ \\
Limited strategies & & & & & - & $0.53^{* *}$ & $0.55^{* *}$ \\
Difficulties impulse control & & & & & - & $0.72^{* *}$ \\
Difficulties goal-directed behaviours & & & & & & - \\
\hline
\end{tabular}

* Correlation is significant at the 0.05 level (two-tailed).

** Correlation is significant at the 0.01 level (two-tailed).

$\mathrm{DSH}=$ deliberate self-harm.

Table 3. Pearson correlations between the number of episodes of DSH at baseline and measures of symptom severity (depression, anxiety and suicidal cognitions) in DSH patients $(n=90)$

\begin{tabular}{lcccc}
\hline & DSH & Depression & Anxiety & Suicidal cognitions \\
\hline DSH & - & $0.28^{* *}$ & $0.26^{*}$ & 0.19 \\
Depression (BDI-II) & & - & $0.53^{* *}$ & $0.74^{* *}$ \\
Anxiety (SCL-90) & & & - & $0.51^{* *}$ \\
Suicidal cognitions (SCS) & & & - \\
\hline
\end{tabular}

* Correlation is significant at the 0.05 level (two-tailed).

** Correlation is significant at the 0.01 level (two-tailed).

$\mathrm{DSH}=$ deliberate self-harm.

BDI-II = Beck Depression Inventory II.

\section{Pearson Correlations between Emotion Regulation Variables and DSH at 9-Month Follow-up}

Correlations between subscales ranged between 0.13 (lack of clarity and DSH) and 0.86 (difficulties with impulse control and difficulties with goaldirected behaviours) (see Table 4). Positive correlations were found between DSH and all of the DERS subscales, except for lack of clarity.

\section{Effects of Treatment on Treatment Outcome (DSH)} (Mediation Test-Step 1)

To test whether treatment condition predicted change in outcome (Path C), we used a linear regression analysis with treatment condition as the predictor variable (coded 0 for TAU and 1 for CBT) and the residual change score of DSH (which is the DSH score at 9-month follow-up corrected for any baseline differences on this measure at baseline) as the outcome variable. Treatment condition predicted change in DSH at 9-month assessment (standardized $\beta=-0.298, p=0.004$ ). Thus, the first requirement for mediation was fulfilled.

\section{Effects of Treatment on the Proposed Mediators (Mediation Test-Step 2)}

To determine whether treatment condition (coded 0 for TAU and 1 for CBT) predicted change in the mediators (Path A), we used separate regression analyses with condition as the predictor variable and the residualized change score of each of the proposed mediators (emotion regulation difficulties and symptom severity) as the outcome variable.

Treatment condition predicted change in emotion regulation difficulties at 9-month assessment: lack of awareness (standardized $\beta=-0.383, p<0.001$ ), lack of clarity (standardized $\beta=-0.438, p<0.001$ ), non-acceptance of emotions (standardized $\beta=$ $-0.398, p<0.001$ ), limited strategies (standardized $\beta$ $=-0.439, p<0.001)$, difficulties with impulse control (standardized $\beta=-0.419, p<0.001$ ) and difficulties engaging in goal-directed behaviours (standardized $\beta=-0.387, p<0.001$ ). In addition, treatment condition predicted symptom severity: depression (standardized $\beta=-0.523, p<0.001$ ), anxiety (standardized $\beta=-0.444, p<0.001$ ) and suicidal cognitions (standardized $\beta=-0.410, p<0.001$ ). Thus, the second requirement for mediation was fulfilled. 
Table 4. Pearson correlations between the number of episodes of DSH at 9-month follow-up and emotion regulation difficulties in DSH patients $(n=90)$

\begin{tabular}{lccccccc}
\hline & DSH & Aware & Clarity & Non-accept. & Strategies & Impulses & Goals \\
\hline DSH & - & $0.30^{*}$ & 0.13 & $0.31^{* *}$ & $0.31^{* *}$ & $0.27^{*}$ & $0.30^{* *}$ \\
Lack of awareness of emotions & & - & $0.62^{* *}$ & $0.54^{* *}$ & $0.57^{* *}$ & $0.47^{* *}$ & $0.47^{* *}$ \\
Lack of clarity of emotions & & & - & $0.62^{* *}$ & $0.64^{* *}$ & $0.71^{* *}$ & $0.58^{*}$ \\
Non-acceptance of emotions & & & & - & $0.81^{* *}$ & $0.76^{* *}$ & $0.75^{* *}$ \\
Limited strategies & & & & & - & $0.81^{* *}$ & $0.82^{* *}$ \\
Difficulties impulse control & & & & & - & $-0.86^{* *}$ \\
Difficulties goal-directed behaviours & & & & & & - \\
\hline
\end{tabular}

${ }^{*}$ Correlation is significant at the 0.05 level (two-tailed).

DSH $=$ deliberate self-harm.

Relationship between Change in the Proposed Mediators and Change in Outcome (Mediation Test-Step 3)

The analyses of Step 3 were performed with the data of the patients who had received the CBT intervention. The data of the patients who received TAU only were not included in these analyses. To determine whether the residualized change scores of the mediators correlated with the residualized change score of DSH (Path B), we used separate linear regression analyses for each of the proposed mediators. Change in difficulties with impulse control predicted change in DSH (standardized $\beta=$ $0.285, p=0.047$ ). Change in difficulties engaging in goal-directed behaviours also predicted change in DSH (standardized $\beta=0.319, p=0.026$ ). However, the other emotion regulation difficulties subscales did not predict change in DSH: lack of awareness (standardized $\beta=0.206, p=0.157$ ), lack of clarity (standardized $\beta=0.100, p=0.492$ ), non-acceptance of emotions (standardized $\beta=0.231, p=0.111$ ) and lack of strategies (standardized $\beta=0.207, p=0.153$ ). Furthermore, the residualized change scores of the measurements of symptom severity did not predict change in DSH either: depression (standardized $\beta$ $=0.018, p=0.900$ ), anxiety (standardized $\beta=-0.006$, $p=0.966$ ) and suicide cognitions (standardized $\beta=0.117, p=0.423$ ). Thus, the third requirement for mediation was only fulfilled for difficulties controlling impulses and for difficulties engaging in goal-directed behaviours.

Effects of Treatment on DSH after Controlling for the Effects of the Proposed Mediators (Mediation Test-Step 4)

To assess whether the predictive effects of condition on the outcome DSH (Path C) were significantly reduced when the mediator was


$*=\mathrm{p}<.05 ; * *=\mathrm{p}<.01 ; * * *=\mathrm{p}<.001$

Figure 2. Intent to treat sample: $N=90$. Path models of mediated intervention effects with deliberate self-harm (DSH) and two of the Difficulties in Emotion Regulation Questionnaire subscales: difficulties with impulse control and difficulties with goal-directed behaviours. Dotted lines indicate that the mediator variable was controlled for. $\Delta=$ change

statistically controlled (Path $C^{\prime}$ ), we used separate regression analyses for difficulties with impulse control and difficulties engaging in goal-directed behaviours.

The residualized change score of DSH was entered as the dependent variable. The residualized change scores of the proposed mediators (difficulties with impulse control or difficulties engaging in goal-directed behaviours) and treatment condition (coded 0 for TAU and 1 for CBT) were entered as predictor variables. Results are presented in Figure 2. Results indicated that the change in DSH was mediated by the change in difficulties with impulse control; condition was no longer a significant predictor of change in DSH (standardized $\beta=-0.208$, $p=0.06$ ) whereas difficulties with impulse control remained significant (standardized $\beta=-0.216, p=$ $0.05)$. The entire mediation model accounted for 
$12.8 \%$ of the change in DSH. However, the difference between $C$ (standardized $\beta=-0.298$ ) and $C^{\prime}$ (standardized $\beta=-0.208$ ) is small (see Figure 2). The results also indicated that change in DSH was mediated by change in difficulties engaging in goal-directed behaviours; condition was no longer a significant predictor of DSH (standardized $\beta$ $=-0.196, p=0.07$ ) whereas difficulties engaging in goal-directed behaviours remained significant (standardized $\beta=0.264, p=0.02$ ). The entire mediation model accounted for $14.9 \%$ of the change in DSH. As for difficulties with impulses, the difference between $C$ (standardized $\beta=-0.298$ ) and $C^{\prime}$ (standardized $\beta=-0.196$ ) for difficulties engaging in goal-directed behaviours is small (see Figure 2 ). The above analyses are based on the intent-totreat sample $(n=90)$. Analyses with the completers sample $(n=73)$ led to similar results for each of the four steps of Baron and Kenny (1986).

\section{DISCUSSION}

Cognitive-behavioural therapy for DSH seems to be an effective intervention for DSH as shown in previous studies (Brown et al., 2005; Palmer et al., 2006; Slee et al., 2008a). Despite the demonstrated efficacy of CBT for DSH, very little is known about the mediating variables that lead to the reduction of $\mathrm{DSH}$. What mediates treatment in this time-limited CBT? The theoretical model of DSH predicts that emotion regulation difficulties are important mediators of treatment change (Slee et al., 2007). Consistent with the mediation hypothesis, our findings show that change in difficulties with impulse control and change in difficulties engaging in goaldirected behaviours partially mediate reduction in DSH. However, although the observed effects formally satisfy the Baron and Kenny conditions for partial mediation, the effects of the mediators are small. This suggests that the mediators are not a sufficient or necessary condition for the treatment effect to occur. Furthermore, only two of the four emotion regulation variables that were expected to mediate change were found to mediate treatment effect. Clearly, there is a need for further testing. Nevertheless, reduction of impulse control difficulties and difficulties engaging in goal-directed behaviours (partially) mediated change. These findings seem to suggest that these specific aspects of emotion regulation could be relevant targets of treatment. The process of CBT might involve the modulation of emotional arousal, reducing impulsivity and facilitating behaviour that is in accordance with desired goals (Gratz \& Roemer, 2004).
Although the CBT intervention consisted of a number of different therapeutic elements to target emotion regulation difficulties, only difficulties with impulse control and difficulties with goal-directed behaviours were found to partially mediate treatment effect. These findings might suggest that the primary focus of the CBT had been on the ability to control behaviour in the context of emotional distress, and not so much on the awareness and acceptance of emotions. The high rate of repetition of self-harm found in the present study sample might have asked for greater use of interventions focusing on behavioural control. Awareness and acceptance of emotions might constitute a worthwhile treatment focus when patients are no longer in crisis. For example, MindfulnessBased Cognitive Therapy is aimed at relapse prevention of DSH when patients are no longer acutely depressed (Williams, Duggan, Crane, \& Fennell, 2006). Future studies could compare an intervention similar to the CBT intervention of the present study with a mindfulness-based intervention in order to investigate whether the effectiveness of CBT and mindfulness-based interventions can be attributed to different underlying mechanisms.

Besides these findings on specific emotion-regulation difficulties, there are some other results that deserve attention. Although the CBT intervention significantly reduced depression, anxiety and suicidal cognitions, these measures of symptom severity did not play a mediating role. Before the introduction of DSH-specific treatments such as dialectical behaviour therapy (Linehan, 1993; Miller, Rathus, \& Linehan, 2007) and cognitive therapy for suicidal patients (Berk et al., 2004), DSH was treated indirectly by treating associated mental disorders, such as depression (Miller et al., 2007). Since the CBT intervention in the present study focused on emotion regulation difficulties associated with repetition of DSH and not on depressive or other disorders, the results of this study seem to indicate that a treatment focusing on emotion regulation difficulties can be used successfully to reduce symptom severity as well as DSH. These findings are consistent with DSH-specific therapies, suggesting that reduction of DSH can best be established by addressing emotion regulation difficulties.

However, when interpreting these results, it must be taken into account that six variables representing emotion regulation and only three variables assessing symptom severity were tested. Therefore, the likelihood of chance positive findings was 
much higher for the first group of variables than for the second. Future studies might include an equal number of each group of variables to rule out the possibility of chance positive findings.

According to the theoretical ideas presented in the introduction, DSH is due to deficits in emotion regulation. Surprisingly, however, the correlations reported in Table 2 show that only half of the emotion regulation variables assessed were significantly related to DSH at baseline and correlations were rather small. Interestingly, 'difficulties with impulse control', one of the variables found to partially meet criteria for mediation, did not show a significant correlation with DSH at all. This raises the question whether this variable can be regarded as a mediator for the reduction in DSH if it is not related to DSH at baseline. The lack of significant correlations is a challenge to the underlying theoretical model. The results might suggest that emotion regulation difficulties are correlated with DSH in the general population or general clinical samples, but do not specifically predict the severity of DSH in samples that all show high levels of DSH. The fact that there are significant correlations between DSH and emotion regulation difficulties post-treatment may be consistent with this view, as there is a greater variance in DSH at this stage.

It is also important to discuss the possible role of third variables. There are a number of variables that might explain some of the treatment effects, such as DSM-IV axis II diagnoses and dose and type of treatment received as part of TAU. However, in the present study, the presence of personality disorders was not assessed with a structured clinical interview. Therefore, personality disorders can not be ruled out as a third variable. With regard to dose and type of treatment received as part of TAU, previous analysis showed that both conditions received a comparable level of psychotherapy, psychotropic medication and psychiatric hospitalizations in TAU (see Slee et al., 2008a). However, we did not record specific types of psychotherapy or psychotropic medication in TAU. Therefore, it is unclear if the conditions were equivalent in this respect and whether TAU has influenced the mediator analyses.

Given the limitations of the present study, there is a need for more studies on the same emotion regulation variables. If future studies will replicate these findings and find stronger evidence for specific mediators, it might be interesting to follow the advice of Kraemer, Wilson, Fairburn, and Agras (2002) and develop treatment enhanced in those components associated with the mediators to see whether we can identify the active ingredients of the intervention (e.g., teaching emotion-regulation skills). The benefits of uncovering mechanisms of change would be considerable.

Although this CBT intervention is based on the theoretical assumption that a reduction in DSH is the result of changes in emotion regulation difficulties, other mechanisms of change may also be at work. For instance, Michel and Valach (2001) suggest that a shared mechanism underlying effective treatments of DSH is a strong therapeutic alliance, which allows for a meaningful discourse about DSH, whereas Bateman and Fonagy (2004) point to the importance of enhancement of mentalization (i.e., the ability to understand and reflect upon one's own and other's internal states and their relationship to behaviours). Given the CBT's emphasis on a strong therapeutic alliance as well as on understanding emotions, either of these may be mechanisms of change of the present CBT intervention. Future research may clarify the exact underlying mechanisms.

There are also some other limitations that deserve attention. First, it remains unclear whether the mediation effects would also have been found in individuals who were excluded from the study. In addition, the study primarily involved young females of Dutch nationality and with a history of chronic DSH. This absence of diversity limits the generalizability of findings. Second, the study does not distinguish between suicidal acts and selfinjury. Therefore, it is unclear if different mediators can be found for these behaviours. Third, sessionby-session assessment of emotion regulation difficulties might have given more insight in maintaining factors of DSH. In addition, more frequent assessments would have allowed us to perform a mediation analysis along the lines of Kraemer et al. (2002). Finally, although the proposed mediators were all measured by reliable and valid self-report questionnaires, their assessment could have been improved by using experimental tests of emotion regulation in addition to self-reports.

In conclusion, a manualized time-limited CBT intervention for DSH changes difficulties with goaldirected behaviours and difficulties with impulse control, which partially mediate reduction in DSH. Furthermore, since depression, anxiety and suicidal cognitions did not seem to play a mediating role, there is further evidence for DSH-specific interventions aimed at decreasing emotion regulation difficulties. However, since the mediation effects are small, there is a need for further testing. 


\section{ACKNOWLEDGEMENTS}

We are grateful to the patients who participated in this study. This study was supported by The Netherlands Organization for Health Research and Development (ZonMw) (contract grant number: 2100.0068).

\section{REFERENCES}

Arrindell, W.A., \& Ettema, J.H.M. (1986). Dutch translation of the SCL-90. Lisse, the Netherlands: Swets \& Zeitlinger.

Baron, R.M., \& Kenny, D.A. (1986). The moderatormediator variable distinction in social psychological research: Conceptual, strategic and statistical considerations. Journal of Personality and Social Psychology, 51, 1173-1182.

Bateman, A.W., \& Fonagy, P. (2004). Psychotherapy for borderline personality disorder: Mentalization based treatment. Oxford: Oxford University Press.

Beck, A.T., Steer, R.A., \& Brown, G.K. (1996a). Manual for the Beck Depression Inventory II. San Antonio, TX: Psychological Corporation.

Beck, A.T., Steer, R.A., Ball, R., \& Ranieri, W.F. (1996b). Comparison of beck depression inventories IA and II in psychiatric outpatients. Journal of Personality, 67, 588-597.

Berk, M.S., Henriques, G.R., Warman, D.M., Brown, G.K., \& Beck, A.T. (2004). A cognitive therapy intervention for suicide attempters: An overview of the treatment and case examples. Cognitive and Behavioral Practice, 11, 265-277.

Bijttebier, P., \& Vertommen, H. (1999). Coping strategies in relation to personality disorders. Personality and Individual Differences, 26, 847-856.

Brown, G.K., Ten Have, T., Henriques, G.R., Xie, S.X., Hollander, J.E., \& Beck, A.T. (2005). Cognitive therapy for the prevention of suicide attempts. A randomized controlled trial. Journal of the American Medical Association, 294, 563-570.

Chapman, A.L., Gratz, K.L., \& Brown, M.Z. (2006). Solving the puzzle of deliberate self-harm: The experiential avoidance model. Behaviour Research and Therapy, 44, 371-394.

Davidson, K., Norrie, J., Tyrer, P., Gumley, A., Tata, P., Murray, H., \& Palmers, S. (2006). The effectiveness of cognitive behaviour therapy for borderline personality disorder: Results from the BOSCOT trial. Journal of Personality Disorders, 20, 450-465.

De Leo, D., Bille-Brahe, U., Kerkhof, A., \& Schmidtke, A. (2004). Suicidal behavior. Gottingen, Germany: Hogrefe \& Huber.

Derogatis, L.R., Lipman, R.S., \& Covi, L. (1973). The SCL90: An outpatient psychiatric rating scale. Psychopharmacology Bulletin, 19, 13-28.

Ebner-Priemer, U.W., Welch, S.S., Grossman, P., Reisch, T., Linehan, M.M., \& Bohus, M. (2007a). Psychophysiological ambulatory assessment of affective dysregulation in borderline personality disorder. Psychiatry Research, 150, 265-275.
Ebner-Priemer, U.W., Kuo, J., Kleindienst, N., Welch, S.S., Reisch, T., Reinhard, I., Lieb, K., Linehan, M.M., Bohus, M. (2007b). State affective instability in borderline personality disorder assessed by ambulatory monitoring. Psychological Medicine, 37, 961-970.

Giesen-Bloo, J., van Dyck, R., Spinhoven, P., van Tilburg, W., Dirksen, C., van Asselt, T., Kremers, I., Nadort, M., Arntz, A. (2006). Outpatient psychotherapy for borderline personality disorder: A randomized trial of schema focused therapy versus transference focused psychotherapy. Archives of General Psychiatry, 63, 649-658.

Gratz, K.L., \& Roemer, L. (2004). Multidimensional assessment of emotion regulation and dysregulation: development, factor structure, and initial validation of the difficulties in emotion regulation scale. Journal of Psychopathology and Behavioural Assessment, 26, 4154.

Gratz, K.L., Rosenthal, M.Z., Tull, M.T., Lejuez, C.W., \& Gunderson, J.G. (2006). An experimental investigation of emotion dysregulation in borderline personality disorder. Journal of Abnormal Psychology, 115, 850-855.

Hawton, K., Zahl, D., \& Weatherall, R. (2003). Suicide following deliberate self-harm: Long-term follow-up patients who presented to a general hospital. British Journal of Psychiatry, 182, 537-542.

Kamphuis, J.H., Ruyling, S.B., \& Reijntjes, A.H. (2007). Testing the emotion regulation hypothesis among self-injuring females. Evidence for differences across mood states. Journal of Nervous and Mental Disorders, 195, 912-918.

Kraemer, H.C., Wilson, T., Fairburn, C.G., \& Agras, W.S. (2002). Mediators and moderators of treatment effects in randomized clinical trials. Archives of General Psychiatry, 59, 877-883.

Leible, T.L., \& Snell, W.E., Jr. (2004). Borderline personality disorder and multiple aspects of emotional intelligence. Personality and Individual Differences, 37, 393-404.

Levine, D., Marziali, E., \& Hood, J. (1997). Emotion processing in borderline personality disorders. Journal of Nervous and Mental Diseases, 185, 240-246.

Linehan, M.M. (1993). Cognitive-behavioral treatment of borderline personality disorder. New York: The Guilford Press.

Linehan, M.M., Comtois, K.A., Murray, A.M., Brown, M.Z., Gallop, R.J., Heard, H.L., Korslund, K.E., Tutek, D.A., Reynolds, S.K., Lindenboim, N. (2006). Two-year randomized controlled trial and follow-up of dialectical behavior therapy vs therapy by experts for suicidal behaviors and borderline personality disorder. Archives of General Psychiatry, 63, 757-766.

Michel, K., \& Valach, L. (2001). Suicide as goal-directed action. In K. van Heeringen (Ed.), Understanding suicidal behaviour: The suicidal process approach to research and treatment (pp. 230-254) Chichester: Wiley \& Sons.

Miller, A.L., Rathus, J.H., \& Linehan, M.M. (2007). Dialectical behavior therapy with suicidal adolescents. New York: The Guilford Press.

Palmer, S., Davidson, K., Tyrer, P., Gumley, A., Tata, P., Norrie, J., Murray, H., \& Seivewright, H. (2006). The cost-effectiveness of cognitive behavior therapy for borderline personality disorder: results from the 
BOSCOT trial. Journal of Personality Disorders, 20, 466481.

Rudd, M.D., Joiner, T., \& Rajab, M.H. (2001). Treating suicidal behaviour. An effective, time-limited approach. New York: The Guilford Press.

Schmidtke, A., Bille-Brache, U., De Leo, D., \& Kerkhof, A. (2004). Suicidal behaviour in Europe. Gottingen, Germany: Hogrefe \& Huber.

Slee, N., Arensman, E., Garnefski, N., \& Spinhoven, Ph. (2007). Cognitive behavioral therapy for Deliberate Self-Harm. Crisis, 28, 175-182.

Slee, N., Garnefski, N., Van der Leeden, R., Arensman, E., \& Spinhoven, Ph. (2008a). Outcomes of a randomized controlled trial of a cognitive behavioral intervention for Deliberate Self-Harm patients. British Journal of Psychiatry, 192, 202-211.

Slee, N., Garnefski, N., Spinhoven, Ph., \& Arensman, E. (2008b). The influence of cognitive emotion regulation strategies and depression severity on Deliberate Self-Harm. Suicide and Life-Threatening Behavior, (in press)
Stone, A.A., Broderick, J.E., Shiffman, S.S., \& Schwartz, J.E. (2004). Understanding recall of weekly pain from a momentary assessment perspective: absolute agreement, between- and within-person consistency, and judged change in weekly pain. Pain, 107, 6169.

Tyrer, P., Thompson, S., Schmidt, U., Jones, M., Knapp, M., Davidson, J., Knapp, M., MacLeod, A., \& Catalan, J. (2003). Randomized controlled trial of brief cognitive behaviour therapy versus treatment as usual in recurrent deliberate self-harm: the POPMACT study. Psychological Medicine, 33, 969976.

Williams, J.M.G., Duggan, D.S., Crane, C., \& Fennell, M.J.V. (2006). Mindfulness-based cognitive therapy for prevention of recurrence of suicidal behavior. Journal of Clinical Psychology, 62, 201-210.

Yen, S., Zlotnick, C., \& Costello, E. (2002). Affect regulation in women with borderline personality disorder traits. Journal of Nervous and Mental Disease, 190, 693-696. 